\title{
Performance of tigh Magnesia Ramming Mixes
}

by Rudolph Smith

$\mathrm{P}$ REFERRED material and practice in the installation of open hearth bottoms are controversial subjects. To add to the complexity of the subject a new factor in the form of high magnesia content ramming mix has been added within the past few years.

The open hearth shop at the Pueblo plant of Colorado Fuel \& Iron Corp. consists of 16 furnaces tapping an average of 110 tons. Normal opcrations call for 48 pet hot metal charge in making steel to specifications ranging from 0.5 to 1.00 pet carbon with 75 pet of the total production being around 0.70 pct carbon because of the company's rail and rail-fastening business. Being located in an agricultural district, much of the scrap is country, auto and bundled grades. These conditions have a definite bearing on open hearth furnace bottom maintenance.

To maintain ingot production at the highest level during the past decade, attention was focussed on bottom delays. Replacing bottoms only as often as is economically practicable does not result in minimizing delays. Experience indicates that furnace bottoms deteriorate rapidly and most shop records will produce evidence where

Rudolph Smith is Open Hearth Superintendent at Colorado Fuel \& Iron Corp., Pueblo, Colo. This paper was presented at the Open Hearth Conference, April 10-12,1950, at Cincinnati.

delays on an old bottom run lower than those on a relatively new one. In this shop of 16 furnaces, 30 new bottoms were put in during the past 20 years. Of these, four were rammed in and the remaining were sintered in magnesite. Because of this experience, there developed the question of what might be the result as regards better furnace bottoms if the magnesia content of the material was increased to the highest degree.

This was first attempted by reducing the amount of slag used for sintering in the magnesite. Records show that 35 pct slag was used in sintering in No. 16 bottom in 1927, while the last sintered in bottom of No. 7 furnace was put in with less than 10 pct slag in 1946, the magnesia content being increased from 56 to 74 pct and the lime content reduced by half to 6 pet.

With thinking on this subject along the lines of a higher magnesia content material, the proposal to try such a mix developed by one of the refractory manufacturers was acceptable. This occurred during the early part of 1947 . The material was tried on a number of minor hot repair jobs with indications that it compared satisfactorily with other materials used for similar purposes. Then followed a number of trials using the material for renewing the tap hole section during the rebuilding of the furnace. These trials also showed the material to be satisfactory. The characteristic of the material to set up hard without being subjected to a fusing temperature was of considerable importance.
The first carload of the high magnesia content ramming mix was purchased in September, 1947, and was used throughout the shop for the same purposes as the regular ramming mixes. Records of delays on tap holes, which were put in with the two kinds of ramming mixes, during a particular period were: No. 15-1:20 and No. 13--2:05 with high MgO; and No. 9-1:20 and No. 11-1:55 with the regular mix. The two mixes performed equally well.

This information, plus the satisfactory performance of the high magnesia ramming mix used to repair banks at slag line during furnace rebuilding, led to the decision to use this material in putting in a new bottom in No. 8 furnace. However, because of the caution for which many open hearth people are noted, it was decided to ram in the banks to final contour, but sinter in magnesite on the flat of the bottom. The first round of magnesite was put in on May 6, and the first heat was tapped on May 10 . There wasn't' very much time nor money saved on this job, but information was still being sought on the performance of the high magnesia ramming mix. During the 96hr period of sintering in the magnesite, the furnace was maintained at the highest temperature the roof could stand. The banks being subjected to this temperature showed no failure.

To compare the performance, records of bank delays on No. 8 and No. 7 furnaces were compiled over a period of 16 months following the installation of the new bottoms, No. 7 being all sintered in magnesite. The bank delays for No. 8 furnace with the high magnesia ramming mix averaged $4.1 \mathrm{~min}$ per heat, while those for No. 7 furnace, with magnesite, averaged $5.2 \mathrm{~min}$ per heat.

Following the steel strike, a new bottom was placed in No. 9 furnace. It was rammed in with high magnesia material and about $3 \mathrm{in}$. of magnesite was sintered in on the flat. A similar job was done on No. 16 furnace, but a regular ramming mix was used. Both furnaces set a production record in January, that for No. 16 being an all-shop individual furnace record. No. 16 operated at a rate of 12:6 tons per $h r$ and No. 9 at a rate of 12 tons per hr, which is not bad performance for furnaces with a hearth area of about 500 sq ft. No. 16 is slightly larger, an end furnace.

Comparing the bottom, bank and tap hole delays for the first roof campaign, those for No. 9 are about 35 pct higher than for No. 16, which averaged $12.2 \mathrm{~min}$ per heat. Even though No. 16 is credited with being of superior design and having better crews, the performance of the high magnesia ramming mix has not equalled that of the regular mix in this particular trial.

Since Mar. 1, three furnaces have been put into operation, the bottoms of which were surfaced with different brands of ramming mixes. One is a high magnesia material. In each case, prior to shutting down for rebuilding, the furnace was washed out, removing about 6 in. off the flat and as much as possible off the banks. 